\title{
Measuring Stress, The First Step towards Understanding It
}

\author{
Jordi Aguiló Llobet* \\ Bioengineering Program, CIBER-BBN, Spain
}

Received: 阱 January 23, 2019; Published: 制 January 30, 2019

*Corresponding author: Jordi Aguiló Llobet, Microelectronics and Electronic Systems Department, Autonomous University of Barcelona, Bellaterra, Spain

Abstract

A strategy and methodology for the quantitative evaluation of stress is proposed in this article. The intrinsic complexity of stress is taken into account.

\section{Introduction}

The Stress is a major concern of the twenty-first century and will be more so in coming decades. It is a key factor in physical and mental illness, including drug abuse and suicide, and also depression, diabetes mellitus, and cardiovascular risk. Stress is dramatically increasing in prevalence owing to the need by individuals to constantly respond to ongoing aggressions and challenges facing them in modern-day society. Recently, the World Health Organization identified stress as a "worldwide epidemic of the twenty-first century," not only because of its direct medical, social, and economic effects, butalso because of its close relationship with co-morbidities, and the pushing up chronic mental and physical illness. Although stress remains a significant modern-day challenge, I am of the opinion, and it probably has an engineering bias, that two main obstacles are significantly and negatively affecting a diagnosis of stress and the ability to optimally follow it up. I am referring to the lack of a precise definition of stress, except in its most severe manifestation, and the lack of a reliable, objective, and repeatable method with which to measure its severity. Without these, it is not practically possible to ensure its effective prevention, either through early identification or subsequent management.

It is also difficult to determine the efficacy of any applied therapies under these conditions. Understanding the concept of stress is intuitive to ordinary people, whereas is open to ambiguity when deliberated by specialists. There is a huge "nobody's land" between pathological stress and "healthy" stress, that is, the amount of stress needed to be happy or the exact dose to be efficient. In addition, a quantitative assessment of stress is not feasible because of the lack of valid biomarkers, a scale, or a measurement methodology easily applicable. The absence of a reliable, noninvasive, objective, and continuous measurement method means that the references that are necessary to ensuring a sound diagnosis and effective management are also lacking.

Although the relationship between certain biomarkers of stress, such as cortisol, adrenocorticotropic hormone ACTH, heart rhythm, and galvanic skin response, has been acknowledged for years, none of these are considered to constitute a gold standard reference owing to the complexity of stress etiology. Recently, other indicators, such as heart rate variability, respiratory abnormalities, neuropeptide $Y$, and inflammatory biomarkers (such as interleukin- 6 or tumor necrosis factor- $\alpha$ ), have also proposed as biological markers of stress. Accurate, reliable, and continuous monitoring of the "instantaneous" stress state of the subject would undoubtedly contribute to enhanced diagnosis and followup. Treatment could be personalized by refining the relationship between medications or other interventions and the subject's state, which, in turn, would contribute to better knowledge of disease causes and treatment-related mechanisms.

\section{Method and Experiments}

Currently, an exponential increase in the computation capacity, as well as paradigm shifts introduced by machine learning or, in general by big data algorithms, are facilitating new approaches in the evaluation of stress (with all its complexities) and health care in general. Deterministic thinking (i.e., seeking and justifying causes and effects) can be combined today with statistical thinking i.e., identifying patterns and justifying conclusions with a high degree of success. When applying pure statistical thinking, it is not necessary to identify the reason for or cause of something; having "enough" data will assure the validity of the results. However, as explained by 
Pedro Domingos [1], "the catch is that enough data could be infinite and learning from finite data requires making assumptions." This could mean, in the present case, that there is a need to preserve the validity of conclusions by limiting the study to a certain type of stress in a specific population. In addition, even if the same descriptor is used (i.e., stress), is it feasible to compare the acute stress experienced by a student moments before an examination with the chronic stress experienced by an informal caregiver?

The path that is proposed [2] and that has already begun seeks to answer the previous question, attempting to obtain useful information from different populations experiencing the effects of moderate stressors. The current study was structured around 11 pilot studies that were conducted on different sample populations

Table 1: The 11 original trials. involving varying types of stress and stressors, applied at different intensities and times. Each of the pilot studies comprised a prospective observational study governed by a specific protocol. The action of a particular stressor, real or artificially induced, was applied to a specific population in each trial. During each session, and in accordance with the different states of the subjects, the action of the stressor was evaluated in terms of electrophysiological, biochemical, and psychometric variables subject to a given protocol. The temporal sequences of the relevant values were also simultaneously collected. The 11 original trials are depicted in Table 1. Analysis was conducted of variations between the established parameters, and of correlations between the selected electrophysiological, biochemical, and psychometric parameters for each subject, group, and different states of the subjects, whenever possible.

\begin{tabular}{|c|c|}
\hline Type of stress & Setting \\
\hline \multirow{4}{*}{ Chronic } & Caregivers of chronic patients at home \\
\hline & Caregivers of terminally ill, oncologic patients \\
\hline & Parents of children undergoing major surgery* \\
\hline & Children diagnosed with ADD** \\
\hline \multirow{5}{*}{ Acute emotional } & Healthy students experiencing social and cognitive stress \\
\hline & Patients preparing for knee arthroplasty \\
\hline & Use of stressing situations from a virtual reality world ${ }^{* * *}$ \\
\hline & Patients with bipolar disorder and depression \\
\hline & Medical doctors treating intensive care patients* \\
\hline \multirow{2}{*}{ Traumatic } & Children undergoing major surgery* \\
\hline & Patients who had a stroke less than 3 hours ago*** \\
\hline
\end{tabular}

Note: *: Data analysis not still completed ${ }^{* *}$ ADD: attention deficit disorder ${ }^{* * *}$ : Experiments still not started.

The methodology used for data analysis in these pilots is inspired in the Apache algorithm [3]. It starts with the selection of a set of variables - psychometric, biochemical and electrophysiological from which more than 60 interesting parameters were extracted, the principal components were identified, and factorial analysis was performed of the entire set of psychometric and biochemical variables assumed that they to constitute the stress markers. Then, based on the already executed analysis [4] under an enough population, a stress scale $(0-100)$ was defined together with a correlation between it and the electrophysiological variables. Following this way, a stress level value could be continuously assigned to the individual. So, we have a non-invasive and validated procedure, for a quantitative and continuous assessment of stress.

\section{Conclusion}

Sound results were achieved from the application of the methodology to the trials carried out. The preliminary results on acute emotional [5], chronic [6], and traumatic stress [7] were published. Moving towards a quantitative assessment of stress, using this or a similar methodology, will help to ensure a better understanding of stress and will facilitate its diagnosis and treatment. To this end, I am happy to collaborate with any research team that wishes to apply this approach to the stress itself or to some other mental illnesses.

\section{References}

1. (2018) The Master Algorithm. Pedro Domingos. Basic Books. Hachette Book Group, New York, USA.

2. Aguiló J, Ferrer Salvans P, García Rozo A, Armario A, Corbí A, et al. (2015) Project ES3: attempting to quantify and measure the level of stress. Rev Neurol 61(9): 405-415.

3. (2013) American Psychiatric Association. Diagnostic and statistical manual of mental disorders, fi fth edition (DSM-5). Arlington, VA: American Psychiatric Association.

4. Garzón Rey JM, Arza A, De la Cámara C, Lobo A, Armario A, et al. (2017) Aproximación a una escala de referencia de estrés emocional agudo. Rev Neurol 64: 529-537.

5. Adriana Arza, Jorge Mario Garzón-Rey, Jesús Lázaro, Eduardo Gil, Raul Lopez Anton, et al. (2018) Measuring acute stress response through physiological signals: towards a quantitative assessment of stress. Medical \& Biological Engineering \& Computin.

6. Sira Aguiló, Esther García, Adriana Arza, Jorge M Garzón Rey, Jordi Aguiló (2017) Evaluation of chronic stress indicators in geriatric and oncologic caregivers: a crosssectional study, Stress 21(1): 36-42.

7. Jorge Mario Garzón Rey, Adriana Arza Valdés, Montserrat Nuevo Gayoso, Jordi Aguiló (2018) Effectiveness of patient empowerment over stress related to knee arthroplasty surgery. Enferm Clin 28(3): 186-193. 


\section{ISSN: 2574-1241}

DOI: 10.26717/BJSTR.2019.13.002458

Jordi Aguiló Llobet. Biomed J Sci \& Tech Res

(C) (P) This work is licensed under Creative

Submission Link: https://biomedres.us/submit-manuscript.php

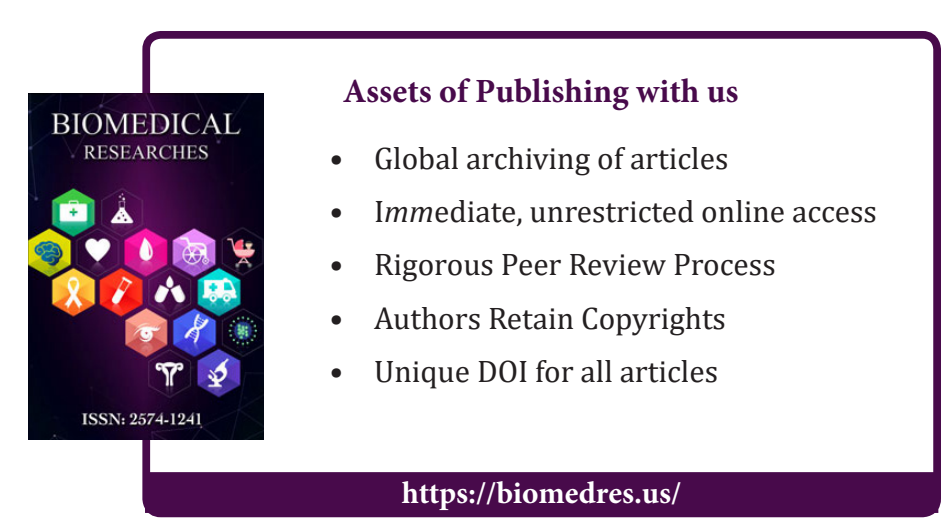

